\title{
Thermally Aware LVCMOS based Low Power Universal Asynchronous Receiver Transmitter Design on FPGA
}

\author{
Amanpreet Sandhu*, Vidhoytma Gandhi, Simranpreet Kaur, Surbhi Huria, \\ Divjot Singh and Wamika Goyal \\ Chitkara University, Patiala - 140401, Punjab, India; \\ Amanpreet.sandhu@chitkara.edu.in, vidhoytma.gandhi@chitkara.edu.in, Simranchhabra2394@gmail.com, \\ Surbhi.huria@chitkara.edu.indivjotdivjot@gmail.com,wamika.goyal@gmail.com
}

\begin{abstract}
Green communication is the latest research trend practiced by researcher in green computing and network communication. There is no extensive work in green UART design. In order to fill this research gap, we are going to design LVCMOS based energy efficient Universal Asynchronous Receiver Transmitter (UART) that will create an avenue for IO standards based green communication. UART (Universal Asynchronous Receiver Transmitter) is a kind of serial communication protocol; mostly used for short-distance low speed, low-cost data exchange between computer and peripherals. Various energy efficient techniques have been applied to the design along with the change in IO standards. It has been concluded that there occurs $97.65 \%$ reduction in clock power, $75.14 \%$ reduction in I/O power, $7.19 \%$ reduction in leakage power, $17.37 \%$ reduction in junction temperature, and $71.12 \%$ reduction in total power dissipation in case SSTL2_II IO/standard, thus it is considered to be most energy and power efficient IO standard to be used in the future.
\end{abstract}

Keywords: Energy Efficient, FPGA, LFM, LVCMOS, Thermal Aware, UART

\section{Introduction}

As shown in Figure 1, A which is sender at one time will behave like receiver at other time and $\mathrm{B}$ will also behaves like sender and receiver both, but at a time they can be either sender or receiver not both.



Sender

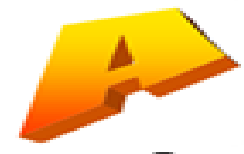

Receiver

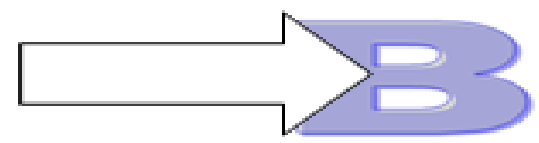

Receiver

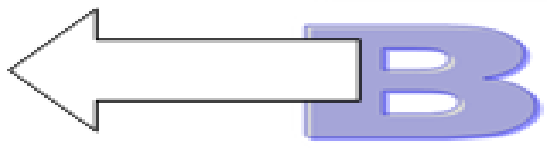

Sender
Figure 1. Anatomy of Communication.
So, we require 2 transmitters and 2 receivers in the communication between A and B as shown in Figure 1 . So we can think of replacing four network devices with only two UARTs (Universal Asynchronous Receiver and Transmitter). UART has two clock signals. It will acts as either receiver or transmitter at once ${ }^{1}$ it will act as receiver when receiver clock is high and act as transmitter when transmitter clock is high. Despite multiple usability in multiple domain of research, there is no extensive work in low power UART design. Low power UART is designed with asynchronous techniques and output load capacitance ${ }^{2,3}$. There is research gap in UART design. There is no LVCMOS IO Standard based UART design yet ${ }^{5,6}$ UART design. In this work, thermally aware ${ }^{9}$ and LVCMOS IO standard based energy efficient design is going to be designed ${ }^{7}$. LVCMOS (Low Voltage Complementary Metal Oxide Semiconductor): LVCMOS

${ }^{*}$ Author for correspondence 
is a widely used switching standard implemented in CMOS transistors. This standard is defined by JEDEC ${ }^{8}$ the LVCMOS standards supported in Virtex-6 FPGAs are: LVCMOS15, LVCMOS18, LVCMOS25, and LVCMOS33 ${ }^{8}$. Frequency Scaling is the technique in which we vary the operating frequency of the device and then change in total power consumption is being analyzed at different frequencies. Energy efficiency can also be achieved by applying another energy efficient techniques such as clock gating, changing the output load, temperature and capacitance ${ }^{10-12}$.

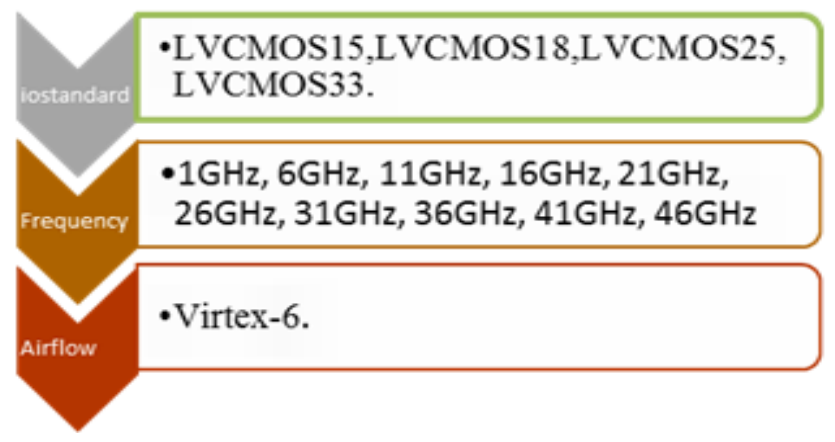

Figure 2. Parameters in Energy Efficient LVCMOS Based UART Design.

\section{Power Analysis}

\subsection{When the Device is being Operated at is LVCMOS15 IO/Standard}

Table 1. Power Dissipation with LVCMOS15 I/O Standard

\begin{tabular}{lccccc}
\hline Frequency & Clock & IOS & Leakage & $\begin{array}{c}\text { Junction } \\
\text { Temp }\end{array}$ & Total \\
\hline $1 \mathrm{GHz}$ & 0.011 & 0.010 & 0.042 & 25.2 & 0.065 \\
$6 \mathrm{GHz}$ & 0.086 & 0.060 & 0.042 & 25.7 & 0.201 \\
$11 \mathrm{GHz}$ & 0.159 & 0.111 & 0.043 & 26.1 & 0.332 \\
$16 \mathrm{GHz}$ & 0.231 & 0.161 & 0.043 & 26.5 & 0.462 \\
$21 \mathrm{GHz}$ & 0.296 & 0.211 & 0.043 & 26.9 & 0.585 \\
$26 \mathrm{GHz}$ & 0.367 & 0.262 & 0.044 & 27.4 & 0.714 \\
$31 \mathrm{GHz}$ & 0.438 & 0.312 & 0.044 & 27.8 & 0.542 \\
$36 \mathrm{GHz}$ & 0.508 & 0.362 & 0.044 & 28.2 & 0.971 \\
$41 \mathrm{GHz}$ & 0.579 & 0.413 & 0.045 & 28.6 & 1.099 \\
$46 \mathrm{GHz}$ & 0.649 & 0.463 & 0.045 & 29.1 & 1.227 \\
\hline
\end{tabular}

From above analysis it is observed that there is $98.30 \%$ reduction in clock power, $97.83 \%$ reduction in IO power, $6.67 \%$ reduction in leakage power, $13.40 \%$ reduction in junction temperature and $94.70 \%$ reduction in total power dissipation when we scale down the frequency from $46 \mathrm{GHz}$ to $1 \mathrm{GHz}$ as shown in Table 1 .

\subsection{When the Device is being Operated at is LVCMOS18 IO/Standard}

Table 2. Power Dissipation with LVCMOS18 IO Standard

\begin{tabular}{lccccc}
\hline Frequency & Clock & IOS & Leakage & $\begin{array}{c}\text { Junction } \\
\text { Temp }\end{array}$ & Total \\
\hline $1 \mathrm{GHz}$ & 0.011 & 0.012 & 0.042 & 25.2 & 0.068 \\
$6 \mathrm{GHz}$ & 0.086 & 0.074 & 0.043 & 25.7 & 0.215 \\
$11 \mathrm{GHz}$ & 0.159 & 0.136 & 0.043 & 26.2 & 0.357 \\
$16 \mathrm{GHz}$ & 0.231 & 0.197 & 0.044 & 26.6 & 0.498 \\
$21 \mathrm{GHz}$ & 0.296 & 0.259 & 0.044 & 27.1 & 0.633 \\
$26 \mathrm{GHz}$ & 0.367 & 0.320 & 0.044 & 27.6 & 0.773 \\
$31 \mathrm{GHz}$ & 0.438 & 0.382 & 0.045 & 28.0 & 0.912 \\
$36 \mathrm{GHz}$ & 0.508 & 0.444 & 0.045 & 28.5 & 1.052 \\
$41 \mathrm{GHz}$ & 0.579 & 0.505 & 0.045 & 28.9 & 1.192 \\
$46 \mathrm{GHz}$ & 0.649 & 0.587 & 0.046 & 29.4 & 1.332 \\
\hline
\end{tabular}

From above analysis it is observed that there is $98.30 \%$ reduction in clock power $97.95 \%$ reduction in IO power, $8.69 \%$ reduction in leakage power, $14.28 \%$ reduction in junction temperature and $94.89 \%$ reduction in total power dissipation when we scale down the frequency from $46 \mathrm{GHz}$ to $1 \mathrm{GHz}$ as shown in Table 2.

\subsection{When the Device is being Operated at is LVCMOS25 IO/Standard}

Table 3. Power Dissipation with LVCMOS25 IO Standard

\begin{tabular}{lccccc}
\hline Frequency & Clock & IOs & Leakage & $\begin{array}{c}\text { Junction } \\
\text { Temp }\end{array}$ & Total \\
\hline $1 \mathrm{GHz}$ & 0.011 & 0.019 & 0.043 & 25.2 & 0.075 \\
$6 \mathrm{GHz}$ & 0.086 & 0.115 & 0.044 & 25.8 & 0.256 \\
$11 \mathrm{GHz}$ & 0.159 & 0.210 & 0.044 & 264 & 0.432 \\
$16 \mathrm{GHz}$ & 0.231 & 0.306 & 0.044 & 27.0 & 0.607 \\
$21 \mathrm{GHz}$ & 0.296 & 0.401 & 0.045 & 27.6 & 0.776 \\
$26 \mathrm{GHz}$ & 0.367 & 0.497 & 0.045 & 28.1 & 0.950 \\
$31 \mathrm{GHz}$ & 0.438 & 0.592 & 0.046 & 28.7 & 1.124 \\
$36 \mathrm{GHz}$ & 0.508 & 0.688 & 0.046 & 29.3 & 1.298 \\
$41 \mathrm{GHz}$ & 0.579 & 0.783 & 0.047 & 29.9 & 1.417 \\
$46 \mathrm{GHz}$ & 0.649 & 0.879 & 0.047 & 30.4 & 1.645 \\
\hline
\end{tabular}

From above analysis it is observed that there is $98.30 \%$ reduction in clock power, $97.83 \%$ reduction in IO power, $8.51 \%$ reduction in leakage power, $17.10 \%$ reduction in junction temperature and $95.44 \%$ reduction in total power dissipation when we scale down the frequency from $46 \mathrm{GHz}$ to $1 \mathrm{GHz}$ as shown in Table 3 . 


\subsection{When the Device is being Operated at is LVCMOS33 IO/Standard}

From above analysis it is observed that there is $98.30 \%$ reduction in clock power, $97.83 \%$ reduction in IO power, $12 \%$ reduction in leakage power, $21.18 \%$ reduction in junction temperature and $95.95 \%$ reduction in total power dissipation when we scale down the frequency from $46 \mathrm{GHz}$ to $1 \mathrm{GHz}$ as shown in Table 4 .

Table 4. Power Dissipation with LVCMOS33 IO

Standard

\begin{tabular}{lccccc}
\hline Frequency & Clock & IOs & $\begin{array}{r}\text { Leakage } \\
\text { Junction } \\
\text { Temp }\end{array}$ & Total \\
\hline $1 \mathrm{GHz}$ & 0.011 & 0.030 & 0.044 & 25.3 & 0.087 \\
$6 \mathrm{GHz}$ & 0.086 & 0.180 & 0.045 & 26.1 & 0.323 \\
$11 \mathrm{GHz}$ & 0.159 & 0.331 & 0.045 & 26.8 & 0.554 \\
$16 \mathrm{GHz}$ & 0.231 & 0.481 & 0.046 & 27.6 & 0.784 \\
$21 \mathrm{GHz}$ & 0.296 & 0.632 & 0.046 & 28.3 & 1.008 \\
$26 \mathrm{GHz}$ & 0.367 & 0.782 & 0.047 & 29.1 & 1.237 \\
$31 \mathrm{GHz}$ & 0.438 & 0.933 & 0.048 & 29.8 & 1.466 \\
$36 \mathrm{GHz}$ & 0.508 & 1.083 & 0.048 & 30.6 & 1.695 \\
$41 \mathrm{GHz}$ & 0.579 & 1.233 & 0.049 & 31.4 & 1.924 \\
$46 \mathrm{GHz}$ & 0.649 & 1.384 & 0.050 & 32.1 & 2.152 \\
\hline
\end{tabular}

\section{Results}

\subsection{Total Power Analysis at Different Io Standards of LVCMOS Logic Family}

Table 5. Total Power Dissipation at Different IOSTANDARD

\begin{tabular}{lcccc}
\hline Frequency & LVCMOS & LCMOS & LVCMOS & LVCMOS \\
& $\mathbf{1 5}$ & $\mathbf{1 8}$ & $\mathbf{2 5}$ & $\mathbf{3 3}$ \\
\hline $1 \mathrm{GHz}$ & 0.065 & 0.068 & 0.075 & 0.087 \\
$6 \mathrm{GHz}$ & 0.201 & 0.215 & 0.256 & 0.323 \\
$11 \mathrm{GHz}$ & 0.332 & 0.357 & 0.432 & 0.554 \\
$16 \mathrm{GHz}$ & 0.462 & 0.498 & 0.607 & 0.784 \\
$21 \mathrm{GHz}$ & 0.585 & 0.633 & 0.776 & 1.008 \\
$26 \mathrm{GHz}$ & 0.714 & 0.773 & 0.950 & 1.237 \\
$31 \mathrm{GHz}$ & 0.542 & 0.912 & 1.124 & 1.466 \\
$36 \mathrm{GHz}$ & 0.971 & 1.052 & 1.298 & 1.695 \\
$41 \mathrm{GHz}$ & 1.099 & 1.192 & 1.417 & 1.924 \\
$46 \mathrm{GHz}$ & 1.227 & 1.332 & 1.645 & 2.152 \\
\hline
\end{tabular}

When we scale down frequency from $46 \mathrm{GHz}$ to $1 \mathrm{GHz}$ as shown in Table 5, there is $94.70 \%$ reduction in total power reduction at LVCMOS15, 94.89\% reduction at LVCMOS18, 95.44\% reduction at LVCMOS25, 95.95\% reduction at LVCMOS33.

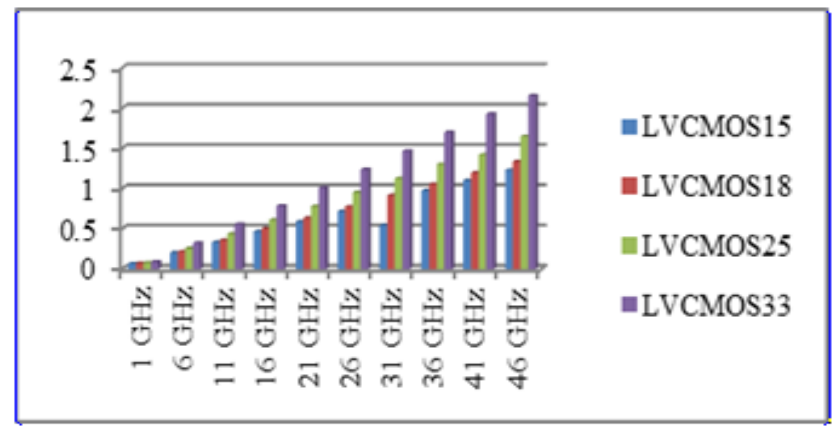

Figure 3. Total Power Dissipation of UART Using Different LVCMOS IO Standards.

\subsection{Junction Temperature Analysis at Different Io Standards of LVCMOS Logic Family}

Table 6. Junction Temperature at Different IO Standard

\begin{tabular}{lcccc}
\hline Frequency & LVCMOS & LVCMOS & LVCMOS & LVCMOS \\
& $\mathbf{1 5}$ & $\mathbf{1 8}$ & $\mathbf{2 5}$ & $\mathbf{3 3}$ \\
\hline $1 \mathrm{GHz}$ & 25.2 & 25.2 & 25.3 & 25.3 \\
$6 \mathrm{GHz}$ & 25.7 & 25.7 & 26.1 & 26.1 \\
$11 \mathrm{GHz}$ & 26.1 & 26.2 & 26.8 & 26.8 \\
$16 \mathrm{GHz}$ & 26.5 & 26.6 & 27.6 & 27.6 \\
$21 \mathrm{GHz}$ & 26.9 & 27.1 & 28.3 & 28.3 \\
$26 \mathrm{GHz}$ & 27.4 & 27.6 & 29.1 & 29.1 \\
$31 \mathrm{GHz}$ & 27.8 & 28.0 & 29.8 & 29.8 \\
$36 \mathrm{GHz}$ & 28.2 & 28.5 & 30.6 & 30.6 \\
$41 \mathrm{GHz}$ & 28.6 & 28.9 & 31.4 & 31.4 \\
$46 \mathrm{GHz}$ & 29.1 & 29.4 & 32.1 & 32.1 \\
\hline
\end{tabular}

When we scale down frequency from $46 \mathrm{GHz}$ to $1 \mathrm{GHz}$ as shown in Table 6, there is $13.40 \%$ reduction in junction temperature at LVCMOS15, $14.28 \%$ reduction at LVCMOS18, 17.10\% reduction at LVCMOS25, 21.18\% reduction at LVCMOS33.

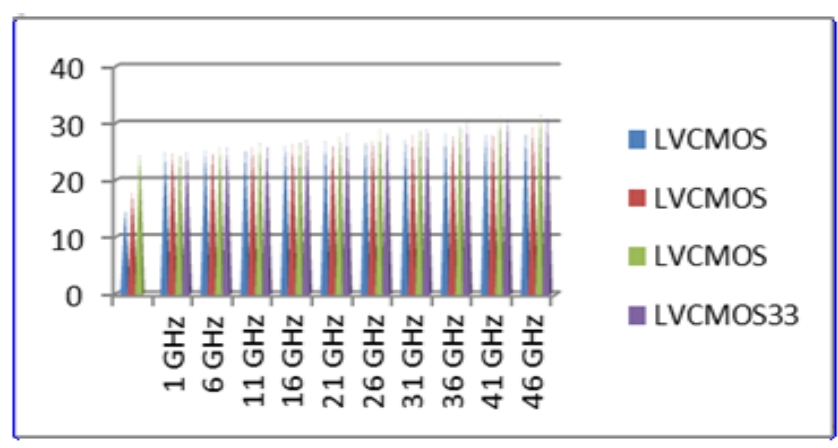

Figure 4. Junction Temperature of UART Using Different LVCMOS IO Standards. 


\section{Conclusion}

The UART is being observed on different-different I/O Standard (LVCMOS). From above analysis, it is observed that changing IO Standard does not have much effect on leakage which is a static power but it is having a significant effect on dynamic power (IO power + Clock power) So we can conclude that there occurs the reduction in total power (Static + Dynamic Power) consumption (Figure 3.) and Junction Temperature (Figure 4.) if we decrease the range of frequency from $46 \mathrm{GHZ}$ to $1 \mathrm{GHZ}$ and if we choose LVCMOS33 IO standard out of all the IO standards of LVCMOS logic family.

\section{Future Scope}

We implement this UART on 28-nm Airtex-7 FPGA. There is a scope to implement this UART on latest $28-\mathrm{nm}$ Kintex-7 FPGA to make the most energy efficient UART. We can also take different frequency ranges other than being used such as Wi-Fi frequencies or frequencies in range of $\mathrm{MHZ}$ or GHZ to make more efficient design of UART.

\section{References}

1. Norhuzaimin J, Maimun HH. The design of high speed UART. IEEE of the Asia-Pacific Conference on Applied Electromagnetics (APCAE'2005); Johor. 2005 Dec 20-21. p. $1-5$.

2. Singh P, Pandey OJ, Pandey B, Das T, Kumar T. Output Load Capacitance Based Low Power Implementation of UART on FPGA. IEEE International Conference on Computer Communication and Informatics (ICCCI); Coimbatore; India. 2014. p. 1-4.

3. Hua, et al. Design and Simulation of UART Serial Communication Module Based on Verilog-HDL [J] Computer and Modernization. 2008; 8:003.
4. Kaur A, et al. Thermal aware energy efficient Gurmukhi Unicode Reader for Natural Language. IEEE Conference of the 9th INDIACom; 2015 Mar 11-13; Chitkara University, Patiala, India. New Delhi, India: IEEE; 2015 Mar 11-13. p. 1524-8.

5. Singla A, Kaur A, Pandey B. LVCMOS Based Energy Efficient Solar Charge Sensor Design on FPGA. IEEE of the 6th India International Conference on Power Electronics (IICPE'2014); NIT, Kurukshetra, India: NIT; 2014. Dec 8-10. p. 1-5.

6. kalia K, Pandey B, Nanda K, Malhotra S, Kaur A, Hussain D M A. Pseudo Open Drain IO Standards Based Energy Efficient Solar Charge Sensor Design on 20nm FPGA. IEEE of the 11th International Conference on Power Electronics and Drive Systems (PEDS’2015); Sydney, Australia. 2015 Jun.

7. Gupta T, Verma G, Kaur A, Pandey B, Singh A, Kaur T. Energy Efficient Counter Design Using Voltage Scaling On FPGA. IEEE International Conference on Communication Systems and Network Technologies (CSNT); Gwalior, India. 2015 Apr 4-6; 5.

8. Virtex-6 Select IO Resources User Guide. Available from:, www.xilinx.com. UG361. 2014 Mar 21; 5.

9. Jain A, Kaur A, Singh S, Pandey B. Thermal Aware Low Power Universal Asynchronous Receiver Transmitter Design on FPGA. IEEE of the 6th International Conference on Computational Intelligence and Communication Networks (CICN); Udaipur, India. 2014 Nov 14-16. p. 939-42.

10. Singh S, Kaur A, Pandey B. Energy Efficient Flip Flop Design Using Voltage Scaling On FPGA. IEEE of the 6th India International Conference on Power Electronics (IICPE); Kurukshetra, India: NTI; 2014 Dec 8-10. p. 1-5.

11. Kaur A, Singh S, Pandey B, Kaur R. Clock Gating Based Low Power Efficient Universal Gurmukhi Unicode Design on FPGA. International Symposium (ICTT'2014); Patiala, India: Chitkara University. 2014 Nov.

12. Kaur A, Singh G, Pandey B, Fazili F, Capacitance Scaling Based Green Gurumukhi Unicode Reader Design for Natural Language Processing. IEEE International Conference on Computing for Sustainable Global Development (INDIACOM), Delhi, India: Bharati Vidyapeeth; 2015 Marc 11-13. p. 1479-83. 